\title{
Commentary on childbirth after mid-urethral sling surgery: effects on long term success and complications
}

\author{
Radhika Patnam ${ }^{1}$ \\ Received: 24 July 2019 / Accepted: 19 August 2019 / Published online: 28 August 2019 \\ (C) The International Urogynecological Association 2019
}

This was a study to evaluate long-term outcomes of a midurethral mesh sling (MUS) placed before one or more childbirths. MUS is one of the most commonly performed urogynecologic procedures, and women often have stress urinary incontinence, but desire continued fertility; in the past, a sling prior to completion of childbearing has not been recommended.

The authors reviewed the National Norwegian Incontinence Registry and found those individuals who had given birth after placement of a MUS. They compared these women to a group of women who underwent MUS without subsequent childbirth at a 1:3 ratio. The two primary outcomes were current subjective SUI cure rate and change in cure rate between those who had children and those who did not at 10 years postoperatively. There were no differences in subjective cure rates between controls and cases. The women in the case group with more than one childbirth after MUS had a significantly lower SUI cure rate at study inclusion compared with the women with one childbirth after MUS $(50 \%$

Radhika Patnam

radhika_patnam@med.unc.edu

1 University of North Carolina at Chapel Hill, Chapel Hill, NC, USA vs. $88 \% ; p=0.006$ ). Risk factors found include BMI $\geq 30$ and repeat surgery for recurrent SUI. Delivery after MUS did not seem to be an independent risk factor for recurrence of SUI symptoms in the regression model.

This study offers long-term data to show that a MUS is a feasible option in women with continued childbearing, especially those who plan only one more child. They did have a long-term follow-up of 10 years and use validated questionnaires, lending greater applicability to their conclusions. The study, however, is a small sampling of only 72 women who had children after MUS placement, of which only 39 had a vaginal delivery, and thus the population size may not have been able to fully capture the true success. Their results do allow for further information to be discussed when a young childbearing woman is making SUI treatment decisions.

Publisher's note Springer Nature remains neutral with regard to jurisdictional claims in published maps and institutional affiliations. 